\title{
Towards optimal personalized diet and vitamin supplementation in NET patients
}

\section{Dear Editor,}

Patients with a neuroendocrine tumor (NET) often have gastrointestinal complaints, leading to impaired absorption of critical food components such as vitamins. The aim of this feasibility study is to explore if 'personalized dietary advice and supplementation of deficient vitamins' (DIVIT) is feasible in NET patients using a SSA to perform a well-powered clinical trial. In this feasibility study, fifteen NET patients using a SSA for over 6 months got the intervention DIVIT. Feasibility was assessed by calculation of participation/dropout rate and number of intervention-related adverse events. Results showed a participation rate of $75 \%$ and dropout rate of $17 \%$. No intervention-related adverse events occurred. We conclude that DIVIT is feasible in NET patients using a SSA. Results from this study will support the development of a well-powered clinical trial.

Patients with neuroendocrine tumors often have gastrointestinal complaints (Pearman et al. 2016). Previously, fat-soluble vitamin deficiencies during long-term somatostatin analogue (SSA) treatment were demonstrated (Fiebrich et al. 2010). Deficient vitamins can lead to complaints. An inverse correlation between vitamin D levels and overall survival (OS) has been observed in 138 gastroenteropancreatic NET patients (Massironi et al. 2017).

NET patients experience a lower quality of life (QoL) as compared with the general population, among others, due to increased bowel movements (Pearman et al. 2016). Strikingly, little is known about how NET patients using a SSA should be best supported with dietary advice or supplementation of vitamins. The aim of this feasibility study is to explore if DIVIT is feasible in NET patients using a SSA. Results from this study will support the development of a well-powered clinical trial.

Eligible participants were adult patients with NET grade 1 or 2, treated at the University Medical Center Groningen (UMCG), receiving a SSA for more than
6 months and were proficient in Dutch. Patients with serotonin-producing tumors and with non-serotoninproducing tumors were included. Patients with a life expectancy of less than 3 months, a history of another malignancy or planned for abdominal surgery were excluded. The study was approved by the medical ethical committee of the UMCG and registered in ClinicalTrials. gov (NCT02481804). All patients gave written informed consent. Measurements were performed at baseline (T0), week 4 (T4) and end of the study (T18). During the first 4 weeks, standard care was continued. Thereafter, patients started with DIVIT. Dietician consultations were conducted at T4 by 1 out-patient visit and after 5, 10 and 15 weeks by telephone. Vitamin supplementation started at $\mathrm{T} 4$ or at week 8 . The intervention, DIVIT, was based on the patients' individual situation and on Dutch Guidelines and provided patients insight in how they could adjust their diet to obtain a healthy diet leading to less gastrointestinal symptoms (Gezondheidsraad 2006). For a vitamin plasma vitamin $K_{1}<0.8 \mathrm{nmol} / \mathrm{L}$, oral phytomenadione once daily $2 \mathrm{mg}$ during 2 months was supplied (Jagannath et al. 2015). For a plasma vitamin A $<0.8 \mu \mathrm{mol} / \mathrm{L}$, retinol $25,000 \mathrm{IU}$ once daily during 2 weeks, followed by 5000 IU once daily was supplied, until sufficient values were reached. Patients with a plasma $25-\mathrm{OHvit} \mathrm{D}$ $<50 \mathrm{nmol} / \mathrm{L}$ were treated with $800 \mathrm{IU}$ cholecalciferol once daily, chronically. Furthermore, if patients had plasma vitamin $\mathrm{E}<19.2 \mu \mathrm{mol} / \mathrm{L}$, alpha-tocopherol acetate $100 \mathrm{mg}$ once daily was supplied, until sufficient values. In case patients had tryptophan in PRP $<40 \mu \mathrm{mol} / \mathrm{L}$, nicotinamide once daily $250 \mathrm{mg}$ was supplied. Vitamin $B_{12}$ was supplied if plasma levels were $<145 \mathrm{pmol} / \mathrm{L}$ with a starting dose of hydroxocobalamin $1000 \mu \mathrm{g} 2$ times a week intramuscular during 1 month, followed by $1000 \mu$ g once in 2 months (Brink et al. 2014).

The primary outcome; feasibility, was assessed by calculation of participation/dropout rate and number of 
intervention-related adverse events. At baseline, patientand illness-related characteristics were collected by examination of the medical records.

QoL was measured with the European Organisation for Research and Treatment (EORTC) questionnaire for gastrointestinal NET (QLQ-GINET21) and for cancer patients the QLQ-C30. General distress and distress from problems were measured with the distress thermometer and problem list (PL). Empowerment was measured with four domains of the Dutch version of the Construct Empowering Outcomes (CEO) questionnaire. The PatientGenerated Subjective Global Assessment (PG/SGA) was used to measure patients' nutritional state.

Vitamin A, D, E, $\mathrm{K}_{1}, \mathrm{~B}_{12}$ in plasma were analyzed. Tryptophan levels in patients and serotonin levels for routine analysis were assessed in PRP samples (Kema et al. 2001). 24-h Urine was used for analysis of 5-hydroxyindolacetic acid and $\mathrm{N}^{1}$-methylnicotinamide $\left(\mathrm{N}^{1}-\mathrm{MN}\right)$, the main metabolite of vitamin $\mathrm{B}_{3}$, (Bouma et al. 2016).

Mean and standard deviation (s.D.) for normal distribution and median and interquartile range for other distributions were calculated. The scores of questionnaires were calculated according to corresponding guidelines. Mean scores were used for each domains of the PL and CEO questionnaire. Effect sizes (ES) for difference in scores on each endpoint of the EORTC questionnaires, PL and PG-SGA, proportion of patients with normal vitamin values, and ES of the outcomes of CEO were determined with Cohen's d. An ES of $0.20-0.50$ is defined as small, between 0.50 and 0.80 as medium and $\geq 0.80$ as large. Analyses were performed with SPSS, version 23 for windows (SPSS).

Twenty-four NET patients were invited to participate between May 2015 and October 2015. Eighteen patients were included and fifteen completed the full study, giving a participation rate of $75 \%$ and a dropout rate of $17 \%$. Baseline characteristics of patients that completed the study are shown in Table 1. Hospital admission occurred in three patients for non-study-related adverse events.

All patients got one or more personalized dietary advices. Nine patients used all dietary advices. One patient said he did not need additional advices. Eleven patients got supplementation of vitamins during the intervention period.

No or only a small effect was found on most items of QoL as measured by the EORTC QLQ-GINET21 and EORTC QLQ-C30. Only the item 'body image' of the QLQ-GINET21 worsened after intervention (ES=0.71). 'Pain', improved after intervention with a medium effect
Table 1 Baseline characteristics $N=15$.

\begin{tabular}{|c|c|}
\hline Characteristic & \\
\hline Age - median (range) & $61(56-69)$ \\
\hline \multicolumn{2}{|l|}{$\operatorname{Sex} N(\%)$} \\
\hline Male & $7(47)$ \\
\hline Female & $8(53)$ \\
\hline \multicolumn{2}{|l|}{ Location primary tumor $N(\%)$} \\
\hline Lung & $2(13)$ \\
\hline Stomach & $1(7)$ \\
\hline Jejunum/ileum & $9(60)$ \\
\hline Unknown & $3(20)$ \\
\hline \multicolumn{2}{|l|}{ Serotonin producing $\mathrm{N}(\%)$} \\
\hline Yes & $13(85)$ \\
\hline \multicolumn{2}{|l|}{ Tumor grade $N(\%)$} \\
\hline Grade 1 & $10(67)$ \\
\hline Grade 2 & $3(20)$ \\
\hline Unknown & $2(13)$ \\
\hline \multicolumn{2}{|l|}{ Prior antitumour treatment $N(\%)$} \\
\hline Somatostatin analogue & $15(100)$ \\
\hline Palliative surgery & $10(67)$ \\
\hline Abdominal surgeryb & $9(60)$ \\
\hline Interferon & $2(13)$ \\
\hline Everolimus & $1(7)$ \\
\hline Radiotherapy & $1(7)$ \\
\hline \multicolumn{2}{|l|}{ Prior use of $\operatorname{diet} N(\%)$} \\
\hline Without consultation of a dietician & $2(13)$ \\
\hline With consultation of a dietician & $3(20)$ \\
\hline Energy enriched diet & $2(13)$ \\
\hline $\begin{array}{l}\text { Diet to without large vessels to prevent } \\
\text { intestinal obstruction }\end{array}$ & $1(7)$ \\
\hline \multicolumn{2}{|l|}{ Prior vitamin use $N(\%)$} \\
\hline Total patients with vitamin use & $6(40)$ \\
\hline Vitamin D & $4(27)$ \\
\hline Vitamin $\mathrm{K}$ & $3(20)$ \\
\hline Vitamin $\mathrm{B}_{12}$ & $2(13)$ \\
\hline Nicotinamide & $3(20)$ \\
\hline
\end{tabular}

aThe NET was defined as serotonin-producing if platelet-rich plasma (PRP) serotonin level $>5.4 \mathrm{nmol} / 109$ platelets or if 5 -HIAA in $24 / \mathrm{h}$ urine

$>3.8 \mathrm{mmol} / \mathrm{mmol}$ creatinine at baseline measurement; btwo patients with abdominal surgery had a hemicolectomy, one patient had a hemicolectomy and sigmoid resection, one patient had a hemicolectomy and a partial small bowel resection and 4 patients had a small bowel resection.

$\mathrm{M}$, mean score; $\mathrm{N}$, number of patients; s.D., standard deviation.

size, and emotional functioning was negatively affected by DIVIT (ES=0.59). The distress thermometer and PL showed an increase in practical problems $(E S=1.17)$ and a decrease in physical problems $(E S=0.79)$ during intervention. Furthermore, the empowerment decreased on the domain 'relation with physician' $(\mathrm{ES}=0.58)$, 'feel better informed' ( $\mathrm{ES}=1.04$ ) and 'confidence in treatment' ( $\mathrm{ES}=0.98)$. A negligible effect $(\mathrm{ES}=0.15)$ was seen on nutrition state measured with the PG-SGA. At the start of intervention, nine patients had vitamin deficiencies. Despite DIVIT, which improved vitamin levels in 5 patients, 7 patients had deficient vitamin levels after the intervention (ES=0.13) (Fig. 1). 

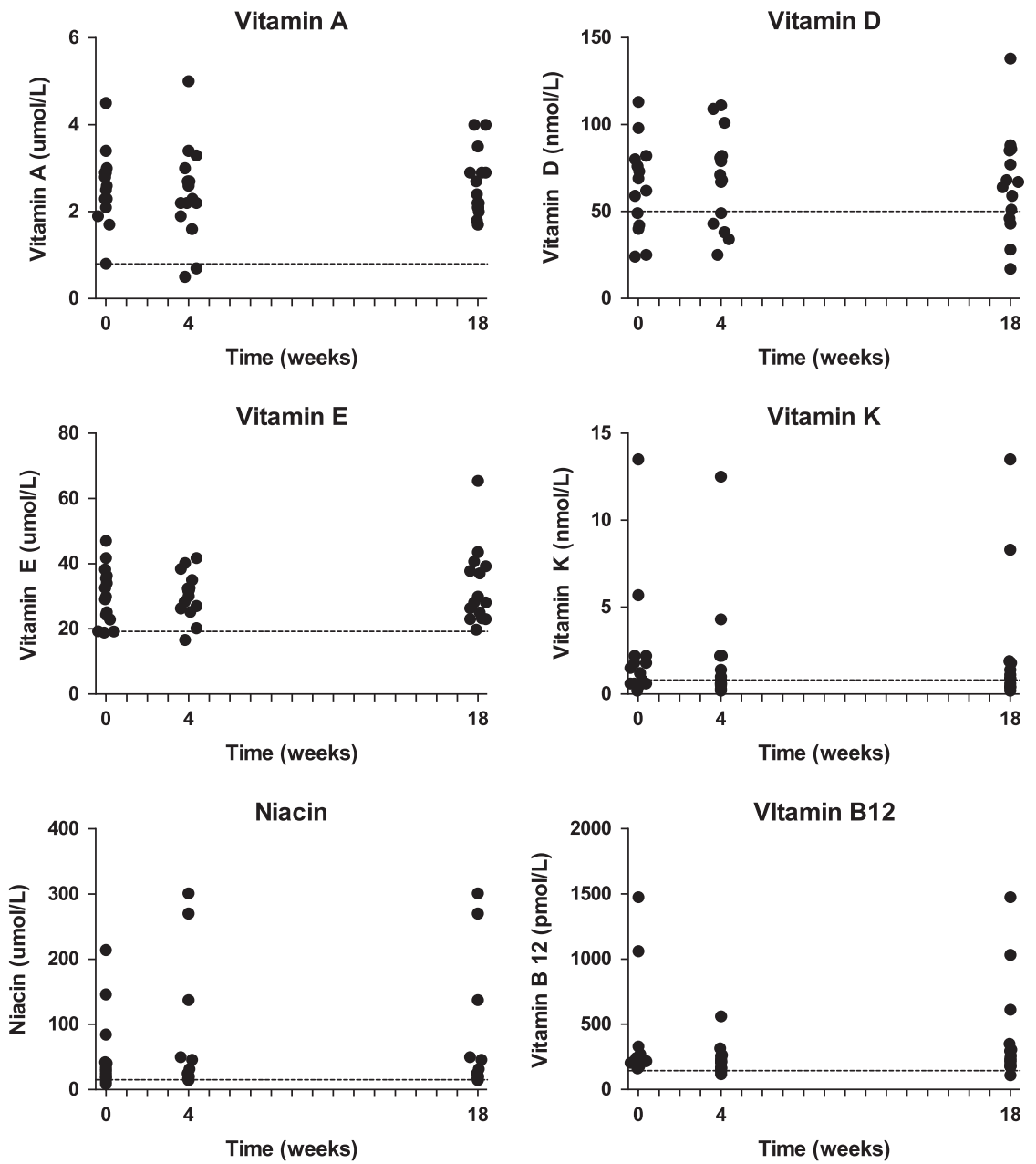

Figure 1

Vitamin values over time. Each dot represents the vitamin value of a patient at the specific time point. The line shows the lower reference value for the specific vitamin.

This is the first prospective feasibility study examining whether a personalized dietary intervention with supplementation of deficient vitamins is feasible in SSA-using NET patients. Several vitamin deficiencies were present at baseline, indicating that standard care might be insufficient. Despite the addition of DIVIT for 14 weeks, which improved vitamin levels in some patients, almost half of patients had one or more deficient vitamin levels after the intervention. This can be due to malabsorption or continuous need instead of temporary use of vitamins. Vitamin supplementation was studied before, like in cystic fibrosis (CF), where patients are at risk for fat-soluble vitamin deficiencies. The restoration of serum vitamin to the normal range after supplementation with vitamins was reported (Jagannath et al. 2015). The doses of vitamin $\mathrm{A}, \mathrm{E}, \mathrm{K}$ and $\mathrm{B}_{12}$ supplementation used in our study were similar with the doses used in other trials; only vitamin $\mathrm{K}$ was supplemented during 8 weeks in our study instead of life long. The vitamin D dose used in our study was based on the required dose for adults without adequate sun exposure and higher than the daily recommended dose by the Institute of Medicine. However, patients with vitamin $\mathrm{D}$ deficiency due to malabsorption probably need higher doses (Lo et al. 1985). Based on our results in combination with the rapid metabolic turnover of vitamin $\mathrm{K}$, we concluded that probably a prolonged duration of vitamin $\mathrm{K}$ supplementation and a higher dose of vitamin D supplementation might lead to restoration of vitamin levels in more patients.

In NET, an association between vitamin D supplementation and OS $(P<0.002)$ was found (Massironi et al. 2017). Furthermore, our intervention showed minimal effect on QoL, as is seen more often in trials measuring the effect of vitamin supplementation in patients with other diseases (Jagannath et al. 2015).

With the results of the current study, it became possible to design a well-powered clinical trial investigating DIVIT for SSA-using NET patients (NCT03143946). Primary endpoint of this single arm intervention trial is the proportion of patients with normal vitamin values, measured with quantitative analysis of blood and urine after the intervention. 
In conclusion, DIVIT is feasible in NET patients using a SSA. NET patients on SSA are at risk for vitamin deficiencies, which are not easily corrected by dietary advices or standard vitamin supplementation. With the results of the current study, it became possible to design a well-powered clinical trial to determine if an intervention with DIVIT leads to a change in proportion of patients with normal vitamin values.

\section{D de Hosson ${ }^{1}$ \\ J Stelwagen ${ }^{1}$ \\ G Bouma ${ }^{1}$ \\ B Sijtema ${ }^{2}$ \\ S Huitema ${ }^{2}$ \\ H J R van Faassen ${ }^{3}$ \\ $\mathrm{G} \mathrm{H}$ de Bock ${ }^{4}$ \\ D J A de Groot ${ }^{1}$ \\ M J E Campmans-Kuijpers ${ }^{5}$ \\ I P Kema ${ }^{3}$ \\ E G E de Vries ${ }^{1}$ \\ A M E Walenkamp ${ }^{1}$}

${ }^{1}$ Department of Medical Oncology, University Medical Center Groningen, University of Groningen, Groningen,

The Netherlands

2Dieticians, Department of Medical Oncology, University Medical Center Groningen, University of Groningen, Groningen, The Netherlands

${ }^{3}$ Department of Laboratory Medicine, University Medical Center Groningen, University of Groningen, Groningen,

The Netherlands

${ }^{4}$ Department of Epidemiology, University Medical Center Groningen, University of Groningen, Groningen, The Netherlands

${ }^{5}$ Department of Gastroenterology, University Medical Center Groningen, University of Groningen, Groningen,

The Netherlands

(Correspondence should be addressed to A M E Walenkamp: a.walenkamp@umcg.nl)
Declaration of interest

The authors declare that there is no conflict of interest that could be perceived as prejudicing the impartiality of this article.

\section{Funding}

This study was supported by an educational grant of Novartis to the UMCG. The sponsor was not involved in any part of the study.

\section{References}

Bouma G, van Faassen M, Kats-Ugurlu G, de Vries EG, Kema IP \& Walenkamp AM 2016 Niacin (Vitamin B3) supplementation in patients with serotonin-producing neuroendocrine tumor. Neuroendocrinology 103 489-494. (https://doi.org/10.1159/000440621)

Brink EA, Breedveld BC \& Peters JAC 2014 Aanbevelingen voor vitamines, mineralen, en spoorelementen. Factsheet. The Hague, the Netherlands: Voedingscentrum. (available at: https://mobiel.voedingscentrum.nl/ Assets/Uploads/voedingscentrum/Documents/Professionals/Pers/ Factsheets/Factsheet\%20Aanbevelingen\%20voor\%20vitamines, \%20 mineralen\%20en\%20spoorelementen.pdf)

Fiebrich HB, Van Den Berg G, Kema IP, Links TP, Kleibeuker JH, Van Beek AP, Walenkamp AM, Sluiter WJ \& De Vries EG 2010 Deficiencies in fat-soluble vitamins in long-term users of somatostatin analogue. Alimentary Pharmacology and Therapeutics 32 1398-1404. (https://doi.org/10.1111/j.1365-2036.2010.04479)

Gezondheidsraad 2006 Richtlijnen goede voeding 2006. Publication no. 2006/21. The Hague, the Netherlands: Gezondheidsraad. (available at: http://www.gezondheidsraad.nl/sites/default/files/200621n.pdf)

Jagannath VA, Fedorowicz Z, Thaker V \& Chang AB 2015 Vitamin K supplementation for cystic fibrosis. Cochrane Database of Systematic Reviews 1 CD008482. (https://doi.org/10.1002/14651858.CD008482. pub4)

Kema IP, Meijer WG, Meiborg G, Ooms B, Willemse PH \& de Vries EG 2001 Profiling of tryptophan-related plasma indoles in patients with carcinoid tumors by automated, on-line, solid-phase extraction and HPLC with fluorescence detection. Clinical Chemistry 47 1811-1820.

Lo CW, Paris PW, Clemens TL, Nolan J \& Holick MF 1985 Vitamin D absorption in healthy subjects and in patients with intestinal malabsorption syndromes. American Journal of Clinical Nutrition 42 644-649.

Massironi S, Zilli A, Bernasconi S, Fanetti I, Cavalcoli F, Ciafardini C, Felicetta I \& Conte D 2017 Impact of vitamin D on the clinical outcome of gastro-entero-pancreatic neuroendocrine neoplasms: report on a series from a single institute. Neuroendocrinology 105 403-411. (https://doi.org/10.1159/000456619)

Pearman TP, Beaumont JL, Cella D, Neary MP \& Yao J 2016 Health-related quality of life in patients with neuroendocrine tumors: an investigation of treatment type, disease status, and symptom burden. Supportive Care in Cancer 24 3695-3703. (https://doi.org/10.1007/s00520-016-3189-z)

Received in final form 20 December 2017

Accepted 9 January 2018 (c) 2018 Society for Endocrinology Published by Bioscientifica Ltd. Printed in Great Britain 